\title{
Low cost microfluidic device for partial cell separation: micromilling approach
}

\author{
Raquel Lopes ${ }^{1}$, Raquel O. Rodrigues ${ }^{1,2}$, Diana Pinho ${ }^{1,3}$, \\ Valdemar Garcia ${ }^{1}$, Helmut Schütte ${ }^{4}$, Rui Lima ${ }^{1,3,5}$, \\ Stefan Gassmann ${ }^{4}$ \\ ${ }^{l}$ ESTiG, Polytechnic Institute of Bragança (IPB), Portugal. \\ ${ }^{2}$ LCM- Laboratory of Catalysis and Materials - Associate \\ Laboratory LSRE-LCM, Faculdade de Engenharia da \\ Universidade do Porto (FEUP), Portugal
}

\begin{abstract}
Several studies have already demonstrated that it is possible to perform blood flow studies in microfluidic systems fabricated by using low-cost techniques. However, most of these techniques do not produce microchannels smaller than 100 microns and as a result they have several limitations related to blood cell separation. Recently, manufacturers have been able to produce milling tools smaller than 100 microns, which consequently have promoted the ability of micromilling machines to fabricate microfluidic devices able to perform separation of red blood cells (RBCs) from plasma. In this work, we show the ability of a micromilling machine to manufacture microchannels with dimensions down to $\mathbf{3 0}$ microns. Additionally, we show for the first time the ability of the proposed microfluidic device to enhance the cell-free layer close to the walls, leading to perform partial separation of RBCs from plasma.
\end{abstract}

Keywords-micromilling; microfluidic device, nonlithographic technique, red blood cells, cell-free layer

\section{INTRODUCTION}

Soft lithography is the most popular method to fabricate microfluidic devices with polydimethylsiloxane (PDMS). The main attraction of this technology is due mainly to its high resolution capabilities and low material cost $[1,2]$. However, the production of the SU-8 moulds by photolithography requires a clean-room environment and specialized equipment that can be quite costly and time consuming. These drawbacks make research progress slow down, especially in institutions without specialized facilities to produce moulds by photolithography. Hence, low-cost nonlithographic techniques to fabricate microfluidic systems are currently being developed. An example is the xurography technique that uses uniquely a cutting plotter machine and adhesive vinyl films to generate the master moulds to fabricate microfluidic channels $[3,4,5]$. Although the xurography is an extremely low cost technique, it is difficult to fabricate microchannels with dimensions smaller than 100 microns. To perform blood cells separation, the microchannels should be smaller than 100 microns $[6,7,8,9]$. Therefore, there is a demand for low-cost nonlithographic methods to produce microfluidic systems with dimensions lower than 100 microns.

\author{
${ }^{3}$ CEFT, Faculdade de Engenharia da Universidade do Porto \\ (FEUP), Portugal. \\ ${ }^{4}$ Jade University of Applied Science, Wilhelmshaven, \\ Germany. \\ ${ }^{5}$ Mechanical Eng. Dep., University of Minho, Campus de \\ Azurém, 4800-058 Guimarães, Portugal.
}

Recently, milling tools manufacturers have been able to produce mills down to 5 microns and consequently have promoted the ability of milling machines to fabricate microchannels smaller than 100 microns without complicated environment such as clean-room. Here, we show the ability of a micromilling machine to manufacture microchannels with 30 microns. Additionally, we present the ability of this microfluidic device to enhance the cell-free layer (CFL) [2, 7, 8] downstream the contraction. In microcirculation the formation of the cell-free layer is caused by the tendency of red blood cells (RBCs) to migrate toward the center of the microchannel, this phenomenon is known as Fahraeus Lindqvist effect $[6,7]$. The presence of this CFL at the regions adjacent to the wall has been used to extract plasma from blood samples [6-9].

The main goal of this work is to show the capability of a microfluidic device, fabricated by a milling process, to perform separation of RBCs from plasma due to the formation of an enhance CFL close to the microchannel walls.

\section{MATERIALS AND METHODS}

\section{A. Geometry of the microchannel}

The microfluidic device tested in this study was fabricated by using a micromilling machine built for micromachining (Minitech Mini-Mill/GX). Fig. 1 shows the geometries and main dimensions of the microfluidic device tested in this study.
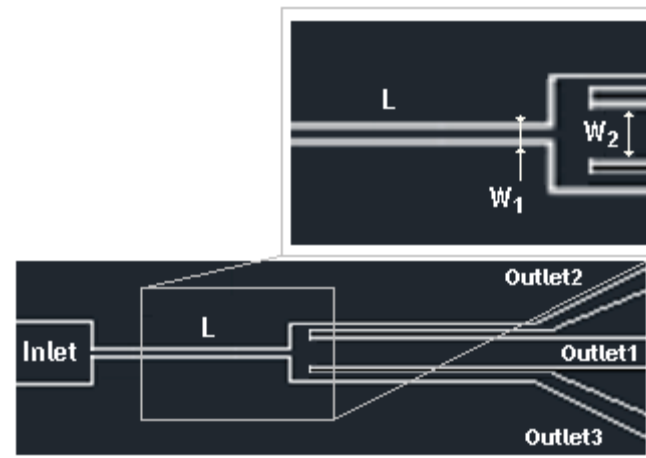

Fig. 1 - Simple contraction microchannel: $\mathrm{W}$ (inlet width) $=300 \mu \mathrm{m}, \mathrm{L}=1300$ $\mu \mathrm{m} ; \mathrm{W}_{1}=30 \mu \mathrm{m} ; \mathrm{W}_{2}=160 \mu \mathrm{m}$. 
The microfluidic device has an inlet width of 300 microns upstream the simple contraction. The contraction has the length (L) of 1300 microns and 30 microns of width $\left(\mathrm{W}_{1}\right)$. Downstream of the sudden contraction, the microchannel has 3 outlets, and the middle one has 160 microns $\left(\mathrm{W}_{2}\right)$.

\section{B. Fabrication of the microfluidic device}

The microchannel geometry was first created using a CAD software, and then fabricated by using a micromilling machine (Fig. 2a) by means of milling tools.

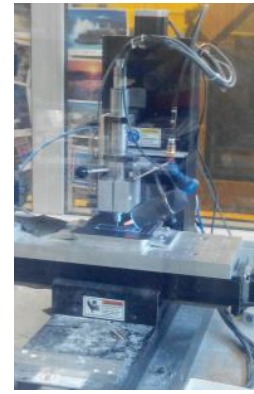

a)

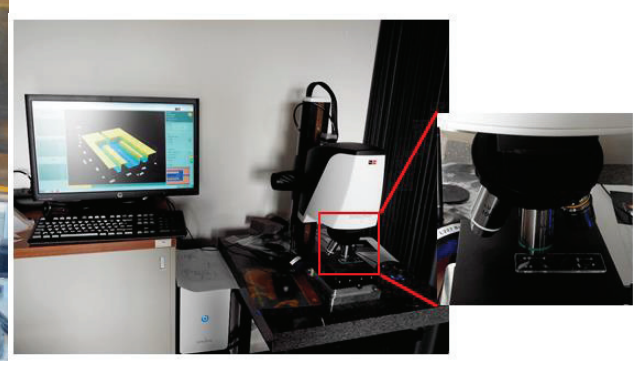

b)
Fig. 2 - a) Micro-milling machine used to fabricate the microchannels. b) 3D optical profiler (Sensofar, plu neox) used to measure the depth of the microchannels.

After drawing the microchannels the Visual Mill software was used to create the $\mathrm{NC}$ code of the milling machine. By using this software, one could define several parameters such as the depth, velocity and angle of the tool. During the fabrication of the device, two different milling tools with the diameter of 30 and $100 \mu \mathrm{m}$ were used.

The milling tools have different heights and the small tools (less than $100 \mu \mathrm{m}$ ) are extremely fragile. Hence, some precautions have to be taken during the fabrication process, such as: start with the middle size tool, make a code for each tool and make the holes for inlets and outlets at the end of the process. A small microscope was used during the fabrication in order to observe the movements of the tools.

The Minitech reads the NC code and performs the milling process. Special attention has to be taken for the z-axis control. The milling machine has no height control to determine the top point of the milled material (acrylic glass). This point, which is the reference point $(\mathrm{z}=0)$ for the milled structure has to be found manually. For this the milling tool will be set in rotation and lowered to the material in $1 \mu \mathrm{m}$ increments. With the help of the microscope, the first touch of the milling tool on the material is observed. This point is stored as a reference point for the $\mathrm{z}$-axis during the machining of one individual part. This procedure has to be repeated for every milling tool and for every new machined part. When the milling process is finish, the acrylic glass was washed with water with detergent and blown with nitrogen to dry the device. After cleaning the device, the depth of the microchannels was measured by means of a 3D optical profiler using confocal and interferometric principle (Sensofar, plu neox) (see Fig. 2b).
The in/outlet part was made by stainless steel tubes with the inner diameter of about $1 \mathrm{~mm}$ with the length of about 1 $\mathrm{cm}$. For assembling, Epoxy glue was used to fix the tubes to the Plexiglass (acrylic glass). Once it got dry, the channel went to an ultrasonic cleaner, with water and detergent, to be washed for about 10-15 minutes. Finally, the microchannels were dried with pressurized air and sealed with an adhesive film (see Fig.3).

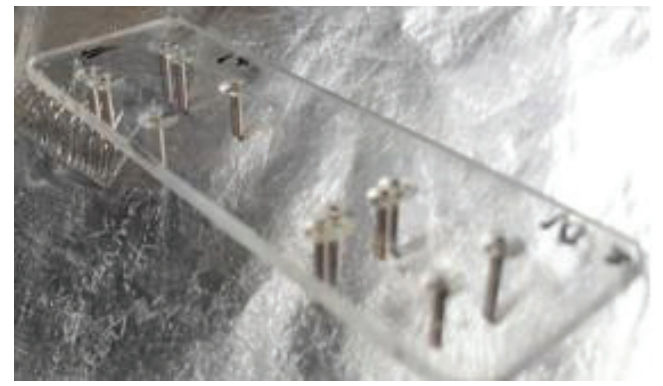

Fig. 3 - Device fabricated by a micromilling machine and sealed with an adhesive film.

\section{Working fluid and experimental set-up}

The working fluid used in the tested microchannel was ovine red blood cells (RBCs) and dextran 40 (Dx40) with 5\% hematocrit (Hct). Briefly, blood was collected from a healthy ovine and heparin was added in order to prevent coagulation. The RBCs were separated from bulk blood by centrifugation ( $2000 \mathrm{rpm}$ for $15 \mathrm{~min}$ at $4^{\circ} \mathrm{C}$ ) and the plasma and buffy coat were removed by aspiration. The RBCs were then washed twice with a physiological saline solution and diluted with Dx40 to make up the required RBC concentration. All blood samples were stored hermetically at $4^{\circ} \mathrm{C}$ until the experiments were performed at room temperature of approximately $20^{\circ} \mathrm{C}$.

The high-speed video microscopy system used in this study is shown in Fig. 4. This system consisted mainly of an inverted microscope (IX71, Olympus) and a high-speed camera (FASTCAM SA3, Photron). The microfluidic device was placed on the stage of the inverted microscope and a syringe pump (Harvard Apparatus PHD ULTRA) was used to produce a constant flow rate.

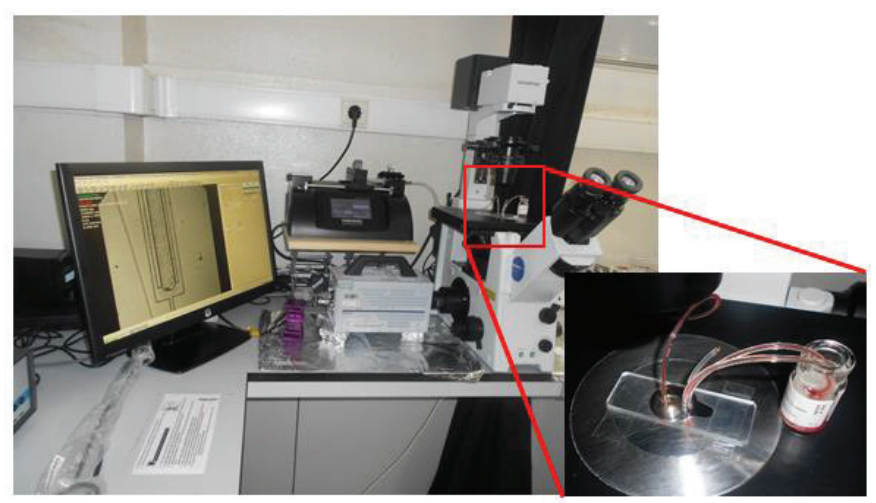

Fig.4 - High-speed video microscopy system used to perform experimental in vitro blood studies. 


\section{Image analysis}

To better visualize the CFL formation in the microchannel and consequently observe the RBCs separation, the captured videos were converted to a sequence of static images (stack) and then, by using " $Z$ project", a function from Image J [9], it was possible to obtain a single image having a sum of all static images. Additionally, a manual tracking plugin (MTrackJ), of the image analysis software ImageJ [9], was used to track individual $\mathrm{RBC}$ flowing around the boundary of the RBCs core. By using MTrackJ plugin, the centroid of the selected $\mathrm{RBC}$ was automatically computed. More detailed information can be found elsewhere $[2,7]$.

\section{RESULTS AND DISCUSSION}

\section{A. Fabrication examination}

To evaluate the milling fabrication, images from the microchannels were obtained by using an SEM (see Fig. 5).
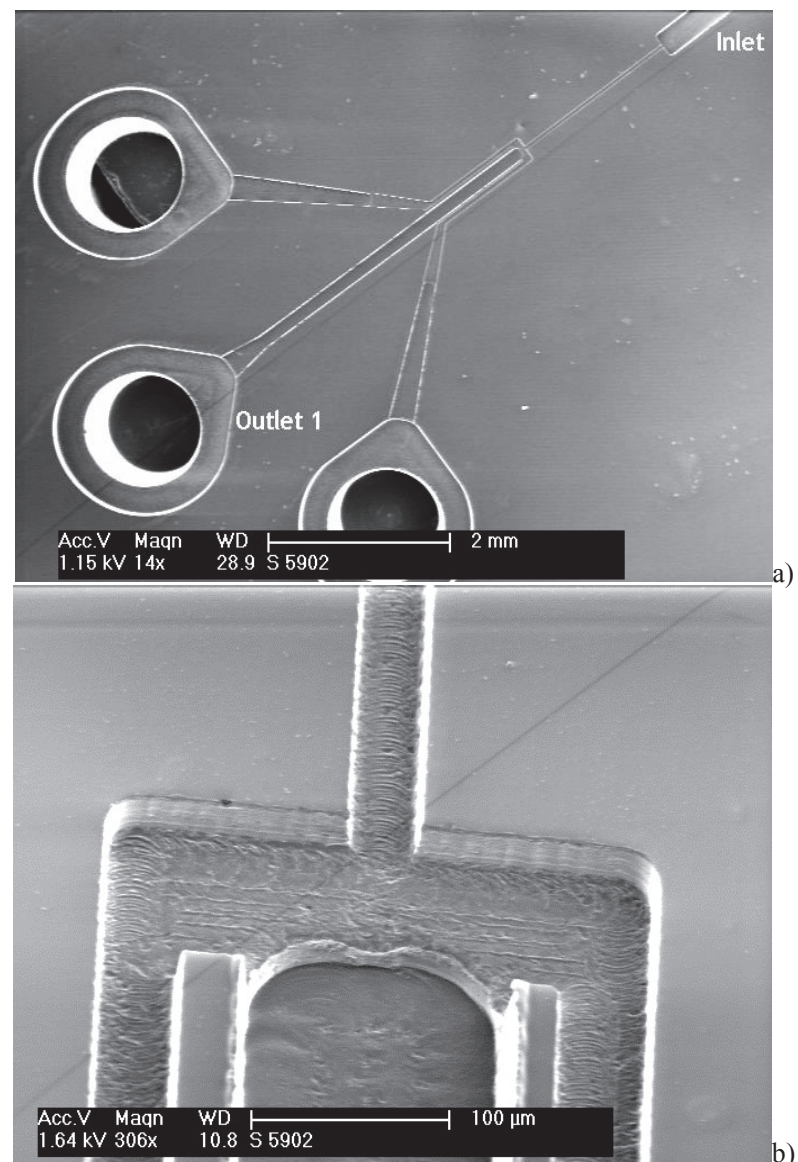

Fig.5 - Qualitative microchannel visualization by using an SEM: a) general view; b) contraction detail.

Additionally the depth of the microchannels was measured by using a $3 \mathrm{D}$ optical profiler. The results obtained by this profiler are presented in Fig. 6.
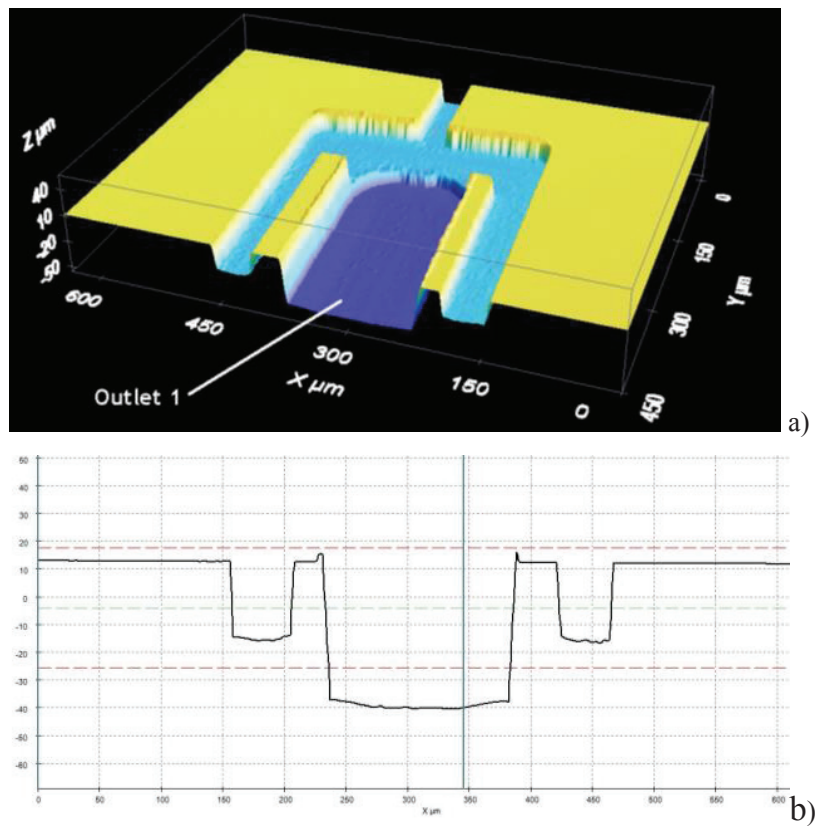

Fig.6 - a) Depth measurements from the 3D optical profiler; b) Graphic representation of the depth of the microchannel.

From Fig. 6 it is possible to observe that the middle walls and depths are not uniform. For instance the depth of the outlet 1 is about $52 \mu \mathrm{m}$ whereas the depth of the other two outlets is about half of the outlet 1 , cf. Fig. $6 \mathrm{~b}$. These results show that by using this microfabrication method it is difficult to obtain an uniform depth along the full length of the microchannel. However, this difference might be an advantage in the cell separation process. Flow experiments are currently being performed in order to evaluate the effect of this parameter in the CFL thickness.

\section{B. Flow visualization}

To evaluate the effect of the contraction in the CFL a sequence of images were obtained both upstream and downstream of the contraction. From these qualitative images, obtained by Image $\mathrm{J}$ (Zproject plugin), it is possible to observe that upstream of the contraction (inlet) there is no CFL (see Fig.7a), while downstream there is a clear CFL close to the both walls (see Fig.7b). These qualitative results show clearly that the contraction enhances the CFL and consequently may result in an efficient separation method.

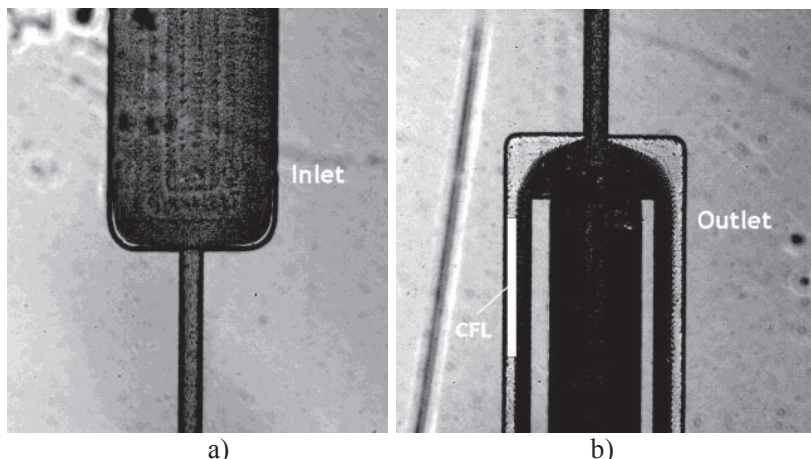

Fig.7 - Qualitative images, obtained by Image J (Zproject plugin): a) upstream of the contraction; b) downstream of the contraction. 
Our high-speed video microscopy system also allows to investigate the motion of individual RBCs flowing within the microchannel. In Fig. 8a represents the tracking of RBCs performed by using the MtrackJ plugin from ImageJ. By tracking individual RBCs around the CFL it was also possible to determine the CFL thickness (see Fig.8b).
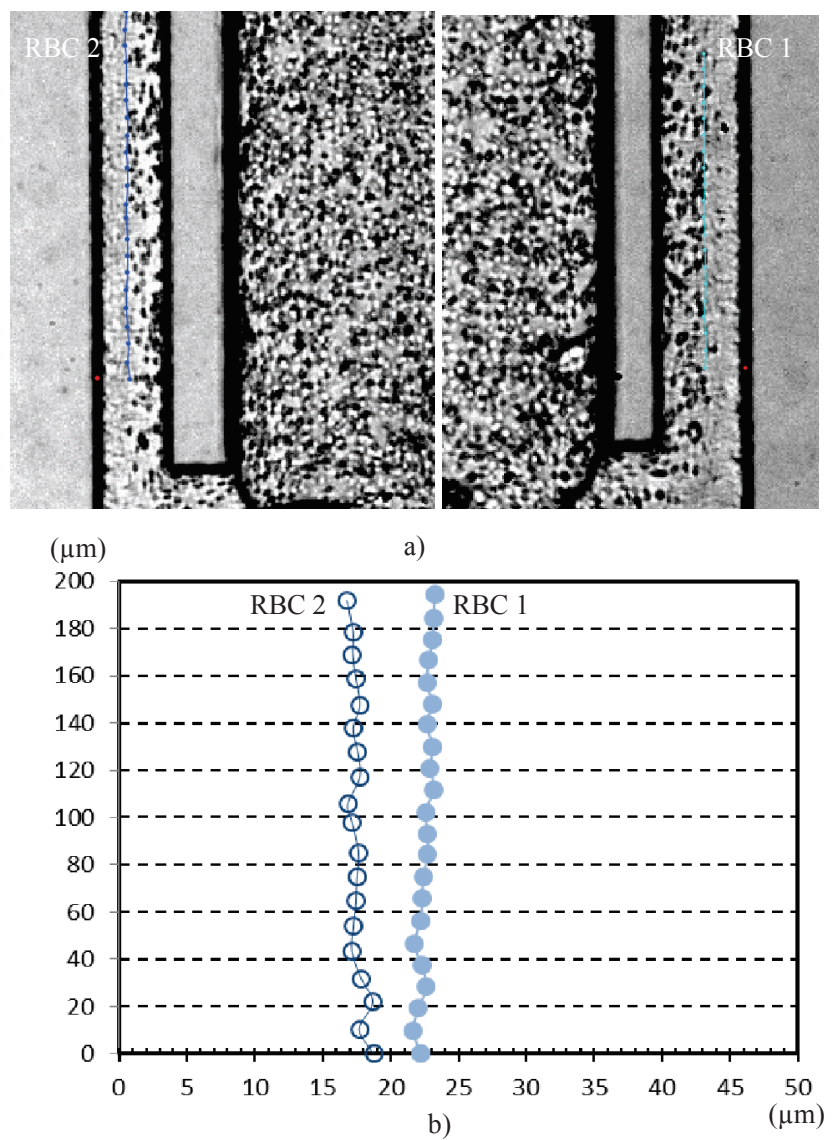

Fig. 8 - a) Tracking of RBCs by using the MtrackJ plugin from ImageJ; b) CFL thickness of two individual RBCs.

The quantitative result from Fig 8b) shows the CFL thickness is around $20 \mu \mathrm{m}$. Moreover, although it is evident that the proposed microfluidic device can increase the CFL downstream the contraction, it cannot perform a full separation of RBCs from plasma, as we can observe in the Fig. 7 and 8, where some RBCs flow through the lateral outlets. A full separation will be obtained if all RBCs flow through the middle outlet.

\section{CONCLUSIONS AND FUTURE WORK}

In this study we can conclude that by using a micromilling machine, it is possible to manufacture microchannels with dimensions down to 30 microns. Additionally, by using a high-speed video microscopy system, we have shown, for the first time, the ability of the proposed microfluidic device to enhance the CFL downstream of the microchannel contraction. In this latter study we have observed that the influence of the proposed geometry, in the enhancement of the CFL, was not enough to perform a full separation of RBCs from plasma. However, we expect that by increasing the length of the contraction and by decreasing the widths of the side walls outlets we can improve the separation efficiency of proposed microfluidic device. Ongoing experiments are currently being performed and results will be published in due time.

\section{ACKNOWLEDGMENT}

The authors acknowledge the financial support provided by PTDC/SAU-ENB/116929/2010 and EXPL/EMSSIS/2215/2013 from FCT (Science and Technology Foundation), COMPETE, QREN and European Union (FEDER). RR and DP acknowledge, respectively, the $\mathrm{PhD}$ scholarships SFRH/BD/97658/2013 and SFRH/BD/89077/2012 attributed by FCT.

\section{REFERENCES}

[1] D.C. Duffy, J.C. McDonald, O.J. Schueller, G.M. Whitesides, "Rapid prototyping of microfluidic systems in poly(dimethylsiloxane)" Anal Chem, vol. 70, pp. 4974-4984, 1998.

[2] R. Lima, S. Wada, S. Tanaka, M. Takeda, T. Ishikawa, K. Tsubota, Y. Imai, T. Yamaguchi, "In vitro blood flow in a rectangular PDMS microchannel: experimental observations using a confocal micro-PIV system", Biomedical Microdevices, vol. 10, pp. 153-167, 2008.

[3] S.O. Sundberg, C.T, Wittwer, J. Greer, R.J. Pryor, O. ElenitobaJohnson, B.K. Gale, "Solution-phase DNA mutation scanning and SNP genotyping by nanoliter melting analysis," Biomedical Microdevices, vol. 9, pp. 159-166, 2007.

[4] E. Pinto, V. Faustino, R. Rodrigues, D. Pinho, V. Garcia, J. Miranda, R. Lima, "A rapid and low-cost nonlithographic method to fabricate biomedical microdevices for blood flow analysis", Micromachines, vol. 6, pp. 121-135, 2015.

[5] D.A. Bartholomeusz, R.W. Boutté, J.D. Andrade. "Xurography: rapid prototyping of microstructures using a cutting plotter", Microelectromechanical Systems, vol.14, pp. 1364-1374, 2005.

[6] M. Faivre, M. Abkarian, K. Bickraj, H.A. Stone, "Geometrical focusing of cells in a microfluidic device: An approach to separate blood plasma," Biorheology, vol. 43, pp. 147-159, 2006.

[7] E. Sollier, M. Cubizolles, Y. Fouillet, J.L. Achard, "Fast and continuous plasma extraction from whole human blood based on expanding cell-free layer devices" Biomedical Microdevices, vol. 12, pp. 485-497, 2010.

[8] D. Pinho, T. Yaginuma, R. Lima, "A microfluidic device for partial cell separation and deformability assessment", BioChip Journal, vol. 7, pp. 367-374, 2013.

[9] T. Yaginuma, M.S. Oliveira, R. Lima, T. Ishikawa, T. Yamaguchi, "Human red blood cell behavior under homogeneous extensional flow in a hyperbolic-shaped microchannel", Biomicrofluidics, vol 7, 54110, 2013.

[10] M.D. Abramoff, P.J. Magalhaes and S.J. Ram, "Image Processing with ImageJ". Biophotonics international vol. 11, pp. 36-42, 2004. 